Scientific Visualization, 2020, volume 12, number 4, pages 98 - 107, DOI: 10.26583/sv.12.4.09

\title{
Visualization of multidimensional surface given in tabular form
}

\author{
Eugene Popovi,A, Anatoly Batiukov²,A, Tatyana Popova3,B, Natalja Vogt4,C, D \\ A Nizhegorodsky State Architectural and Civil Engineering University \\ B National Research University Higher School of Economics \\ c University of Ulm (Germany) \\ D Lomonosov Moscow State University \\ ${ }^{1}$ ORCID: 0000-0002-3058-2369, popov eugene@list.ru \\ 2 ORCID: 0000-0002-1050-1883, absterno@gmail.com \\ 3 ORCID: 0000-0002-1351-222X, tatpop@list.ru \\ 4 ORCID: oooo-0oo1-7190-5702, natalja.vogt@uni-ulm.de
}

\begin{abstract}
The paper describes a graphic model named Exidiagraph. The model is intended for visualization of tabulated hypersurfaces dimension $5 \mathrm{D}$ and higher. Visualization of such hypersurfaces is important for a variety of reasons such as quick and adequate understanding of the physical, chemical, and other phenomena meanings. For example, an important task in physics or chemistry is to find the extrema of hyperfunctions obtained during quantum mechanical calculations. In addition, it seems promising to use the model to solve the problem of finding the path with the least energy between the local extrema of the multidimensional hypersurface. The Exidiagraph model consists of a circular set of planes associated with 2D coordinate systems. The model is a further development of the idea underlying the Lumigraph model. Besides, the visualized Exidiagraph model is aesthetically attractive and has a high degree of visibility.
\end{abstract}

Keywords: Scientific visualization, multidimensional function, hypersurface.

\section{Introduction}

The term scientific visualization (SV) denotes the visual analysis of research data and the process deploys the computer graphics techniques. Being a modern scientific branch SV presents the numerical data of scientific research in the form of visual images that facilitate the information analysis and the exchange of this information. Scientific visualization makes it possible to see the hidden processes of scientific experiments. In other words, scientific visualization makes visible the invisible phenomena. [1-3] The need to visualize invisible phenomena existed long before the emergence of computer technology. The main part of all scientific experiments in physics, chemistry, fluid and gas mechanics, and the theory of elasticity had the goal of not only measuring the quantitative characteristics of the phenomena, but also observing them visually [4-6].

In practice, physical or numerical experiments (calculations) lead to multidimensional functions in a tabular form. Visualization of tabulated multidimensional hypersurfaces is a serious scientific problem. In these cases, it is necessary to solve the problem of mapping multidimensional data to the form of three-dimensional geometric models and visualizing them by computer graphic methods. Generally, visualization of the three-dimensional surface is carried out by NURBS approximation and is not labour-consuming. NURBS curves and surfaces are widely deployed in many Computer Aided Design (CAD) applications for representing the form of cars, aircraft, ships, shoes and numerous other items with sculptured features [7]. 
They are parametrically defined polynomial entities, thus well suited for software implementation, whilst their rational nature provides additional degrees of freedom compared to their non-rational counterparts. The representation exactly reproduces sphere, conic and other special surfaces. NURBS have remained a very popular representation for curves and surfaces in CAD software.

Many factors may have contributed to their success including their versatility, thereby allowing CAD models to be transferred between different systems easily. Studies on other spline forms have been published subsequently, but none have surpassed NURBS in terms of their universal support, despite any advantages they may offer. To visualize $4 \mathrm{D}$ function the authors usually apply volumetric modeling methods to represent solid shapes rather than surfaces. Doing so enables richer simulation, both for dynamics and for illumination in the presence of translucency. The volumetric models are represented by voxels as described, for example, by James Foley [8]. The division of the area of function definition naturally lends itself to a regular grid, making for straightforward representations, and is also easy to build a hierarchy form. This representation is known as a voxel model. In volumetric modeling an object is represented as a collection of voxels in 3D arrangement which may be regular or irregular. Cubic voxels inside a uniform grid aligned with the coordinate axes is the simplest and commonly used representation. It is very common for fluid flow simulation and medical or geoscientific imaging, where the underlying source data are often captured on a regular grid.

In the work [10], we have proposed a method for rendering of a tabulated $5 \mathrm{D}$ hypersurface that is a result of quantum-mechanical calculations of molecular energy. However, the problem of 6D and higher hypersurfaces rendering remains open. This article is dedicated to solving this problem.

\section{Rendering 5D hypersurface}

In work [9-11] we have supplemented Lumigraph model with an additional 2D plot to display a $5 \mathrm{D}$ hypersurface. The main idea here is to map the entire Lumigraph (see [12-13]) to it. The shape of 2D plot should be efficient enough so that the entire surface is visible to the user. This approach is to place the third plane parallel to two initial ones inside the Lumigraph so as to find the points of intersection of line segments with it. The authors called it the additional screen concept. One can see the complete $2 \mathrm{D}$ mapping of the whole $5 \mathrm{D}$ hypersurface in the form of raster image (see Fig. 1) at different locations of the additional screen.
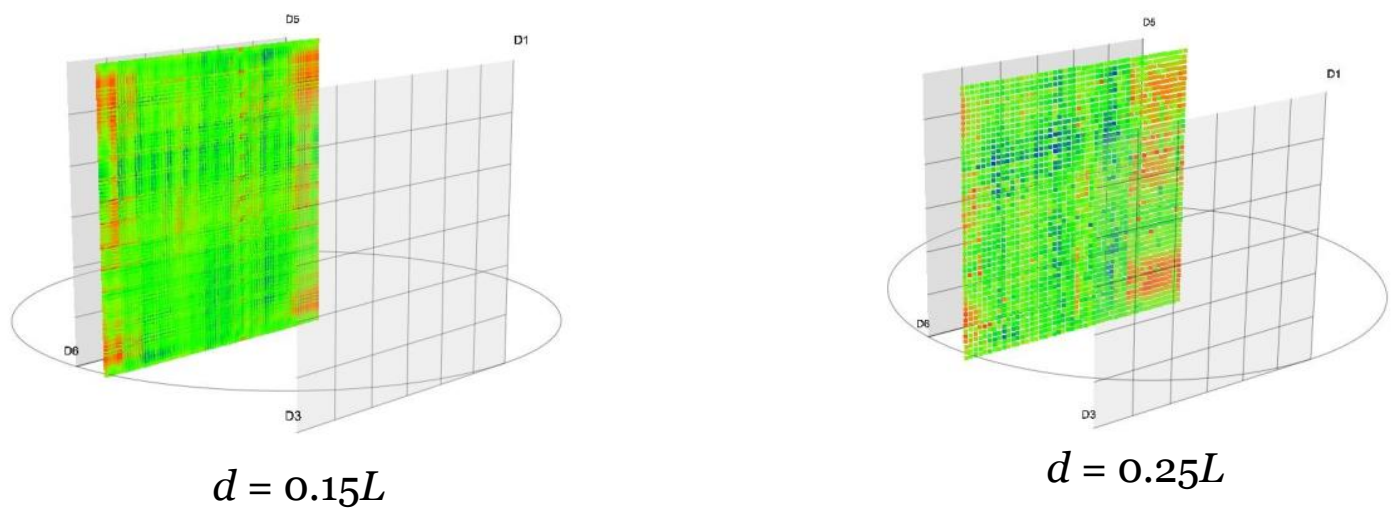


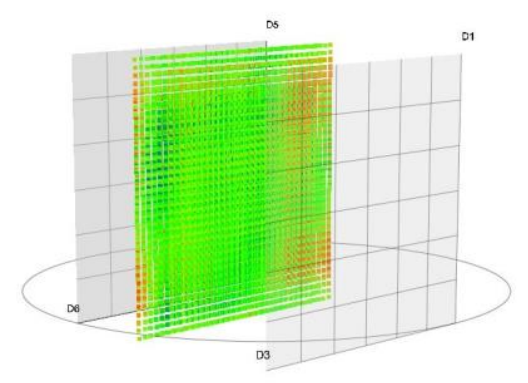

$d=0.35 L$

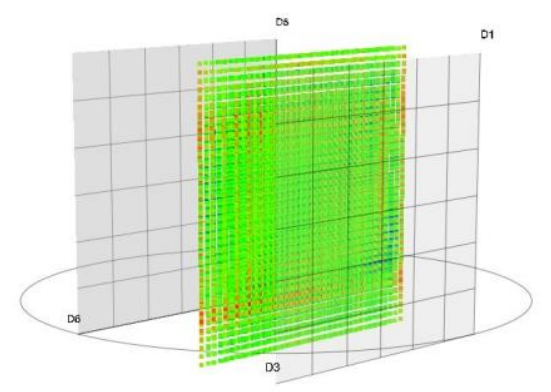

$d=0.65 L$

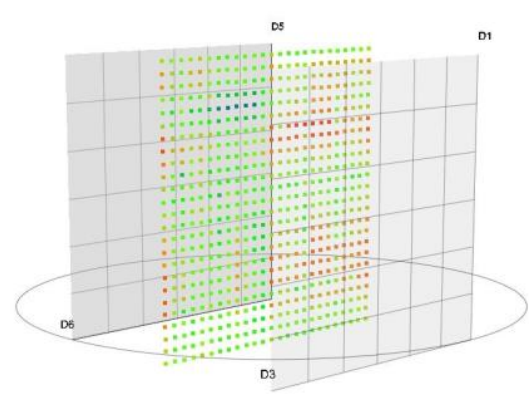

$d=0.5 L$

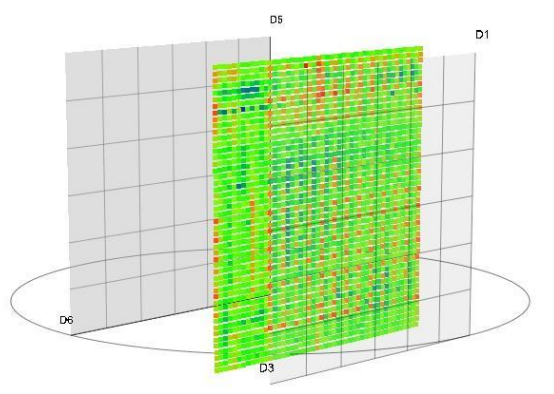

$d=0.75 L$

Figure 1. The additional screen located at different distances inside Lumigraph

The placement of the additional screen was selected empirically by locating it at different distances from the coordinate planes. Fig.1 shows that the worst version of the hypersurface image with its minima is observed when the screen is placed exactly in the middle between the coordinate planes. The best image can be achieved when the screen is placed at a distance $d=$ $0.25 L$, where $L$ is the distance between the coordinate planes.

\section{Rendering 6D-7D hypersurfaces}

We developed a model called Exidiagraph to render $5 \mathrm{D}, 6 \mathrm{D}$, and $7 \mathrm{D}$ surfaces defined in tabular form. A surface the dimension of which depends on the number of variables is often defined as a multidimensional table. The ability to display such hypersurface in space with a number of independent variables equal to 5 or more significantly increases the efficiency of analyzing the results of relevant experiments or calculations.

Mapping a point in a space of dimension that is equal or greater than 6 , is feasible using the idea implemented in the Lumigraph model and described in [9-11]. As mentioned in these works, two parallel planes were used to visualize a point in 4D space, in each of which two 2D coordinate systems were defined. Both planes in the aggregate made it possible to uniquely determine the straight-line segment between them, which was a model of a four-dimensional point. This model of visualization, by analogy with model described in work [12], was called the Lumigraph model and used to visualize $5 \mathrm{D}$ hypersurface minima. 


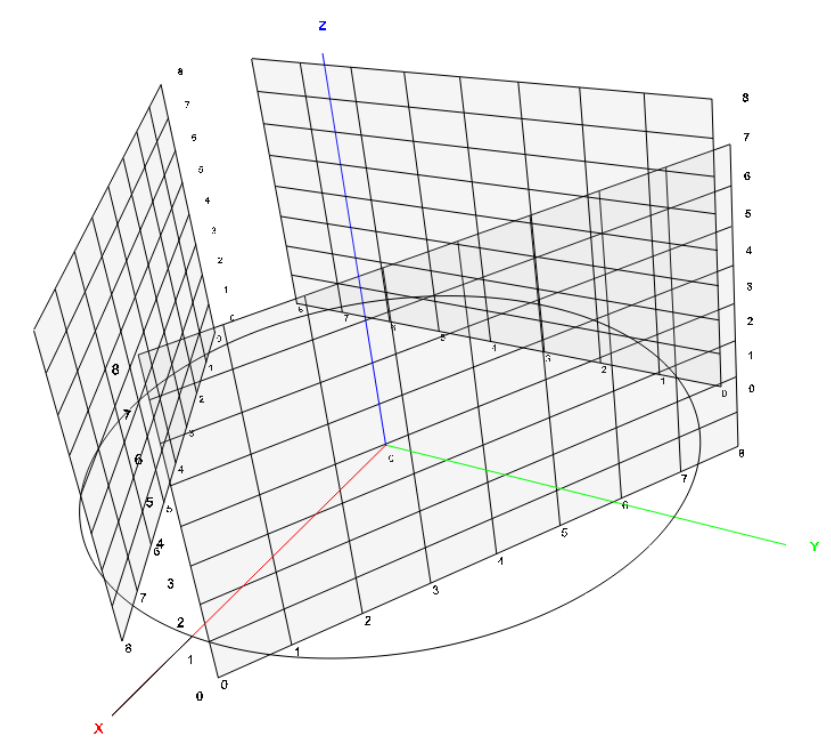

Figure 2. Exidiagraph space

To evolve this idea, a new model was developed to model a point in $5 \mathrm{D}$ (and higher) space and render it. The reconstruction of the Lumigraph model was aimed at adding another plane to it with 2D coordinate system, supplementing the model to a space of dimension 6D (Fig. 2).

Thus, we succeeded in obtaining a coordinate system consisting of three coordinate planes arranged in such a way that all planes form a triangle around the $\mathrm{Z}$ axis. We call this model Exidiagraph (Greek éxi diastáseon gráfima - 6D graph).

To display the points of the studied hypersurface in the Exidiagraph, the set of coordinates can be divided into pairs. For the case of six variables, we can get three pairs of coordinates, one pair for each of the three planes. The sequence of coordinates in pairs can be arbitrary. We can use a similar approach to visualize spaces of greater dimension, for example, 8D, 10D, etc., by breaking the coordinates in pairs and adding a coordinate plane for each additional pair of coordinates. One can associate a point with each coordinate plane, corresponding to one coordinate pair $(\mathrm{x}, \mathrm{y})$. Considering the points obtained on the coordinate planes the vertices of the triangle are depicted in Fig. 3.

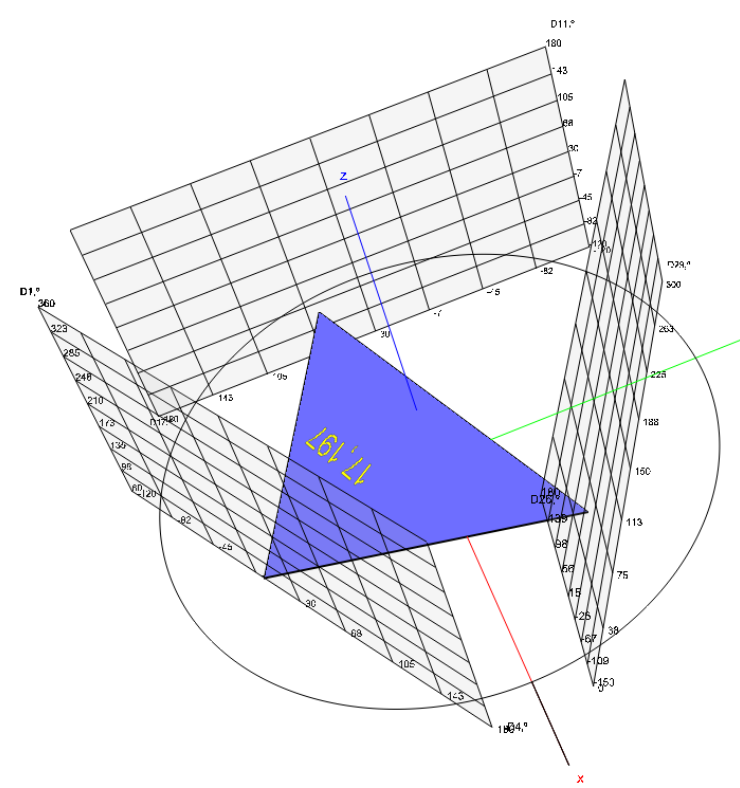

Figure 3. A point in $6 \mathrm{D}$ space rendering in the form of a triangle. 
Thereby, such triangle uniquely corresponds to a point defined by six independent variables. If we associate a certain function value with this triangle and display it in the form of the color associated with the color legend, then we obtain a display of a point depending on six independent variables in 6D space. However, since in the geometric sense, a surface of any dimension can be considered as an infinite point set, its entire visualization by the described model is a considerable challenge. To illustrate it, Fig. 4 shows a visualization of only a few surface points, whence it is clear that even in the case of a limited number of multidimensional points, the picture becomes difficult to perceive.

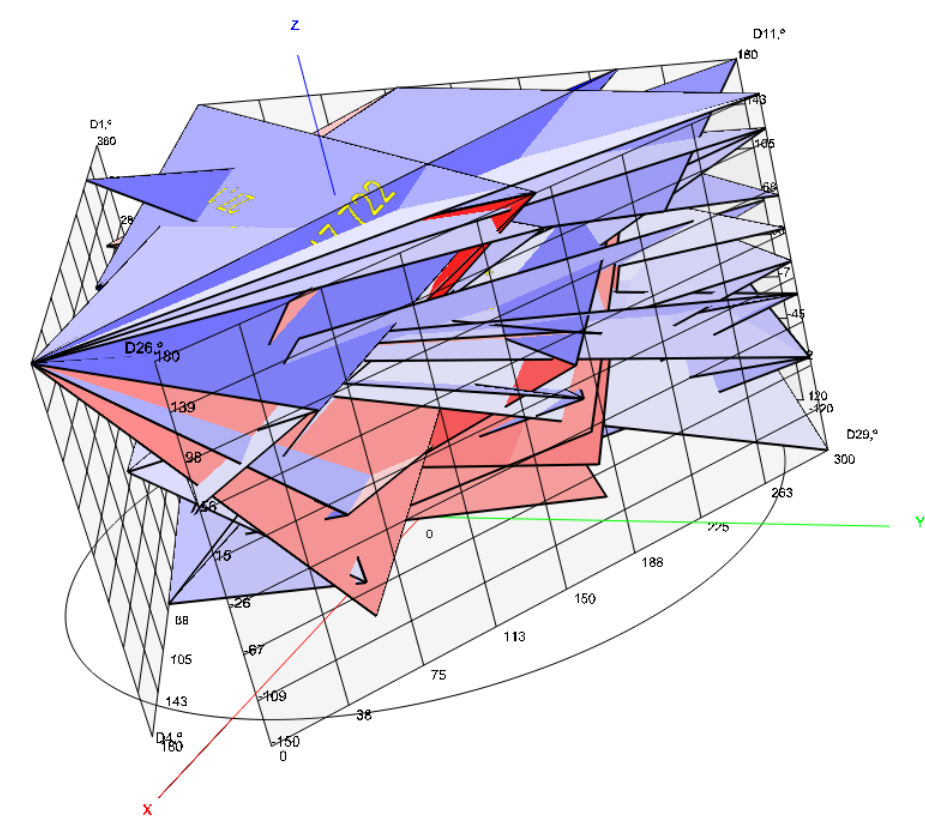

Figure 4. Display of some hyper surface values by triangles.

We propose the following intermediate approach to overcome some perception problem. A point in 6D space is associated with the triangle centroid. The correspondence to its specific coordinates is established by segments of straight lines connecting the centroid with points on the coordinate planes. These segments are called communication lines. If we carry out the visualization of centroids (entire given points of hypersurface) by a set of volume elements, then a clear picture can be obtained, for example, see Fig.5. The term hyper-voxel is assigned to such volume element. We build a color legend based on the tabulated function value. In accordance with this legend hyper voxels are painted in the desired color. The color legend assumes a color change from blue to red, i.e. blue corresponds to the minimum value of the function, while red corresponds to the maximum value. In order to avoid picture cluttering in Fig. 5 communication lines are conditionally hidden.

Further evolution of the Exidiagraph model is associated with giving greater visual clarity to the mapping of a hypersurface. The idea is to normalize the position of the entire centroids along the $\mathrm{Z}$ axis according to the function value at these points as shown in Fig.6, which shows a scheme of such procedure to display a point image in $6 \mathrm{D}$ space. 


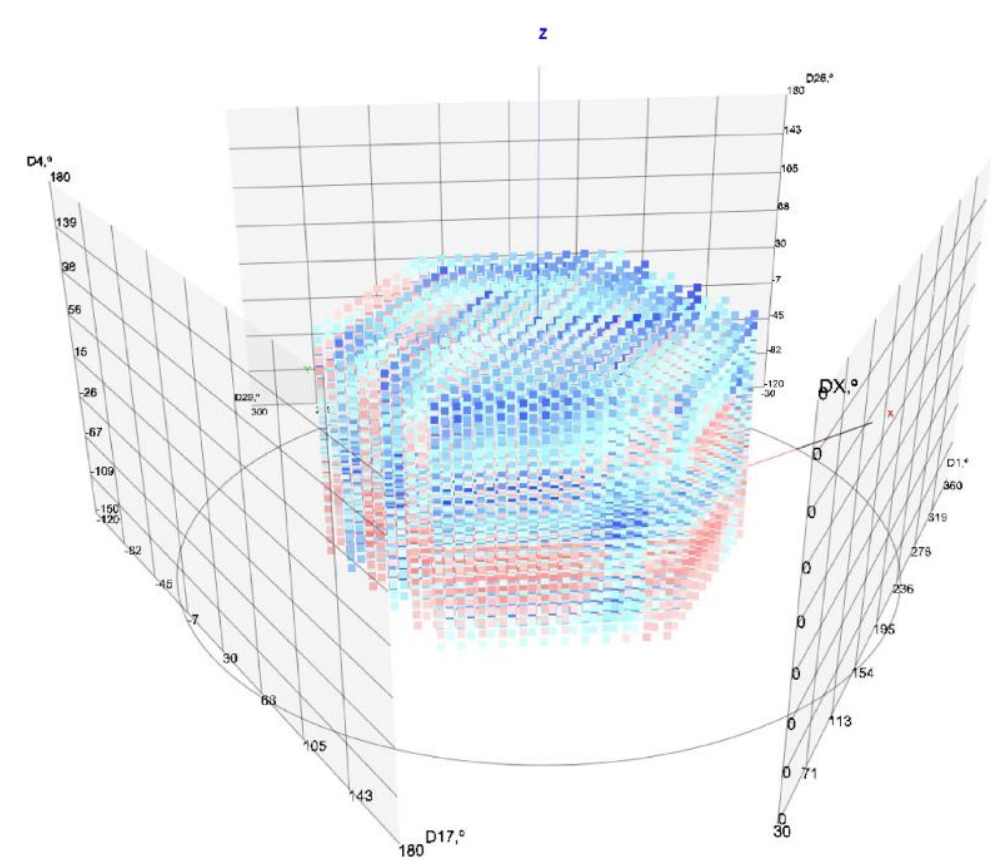

Figure 5. Display of $7 \mathrm{D}$ hyper surface by hyper-voxels.

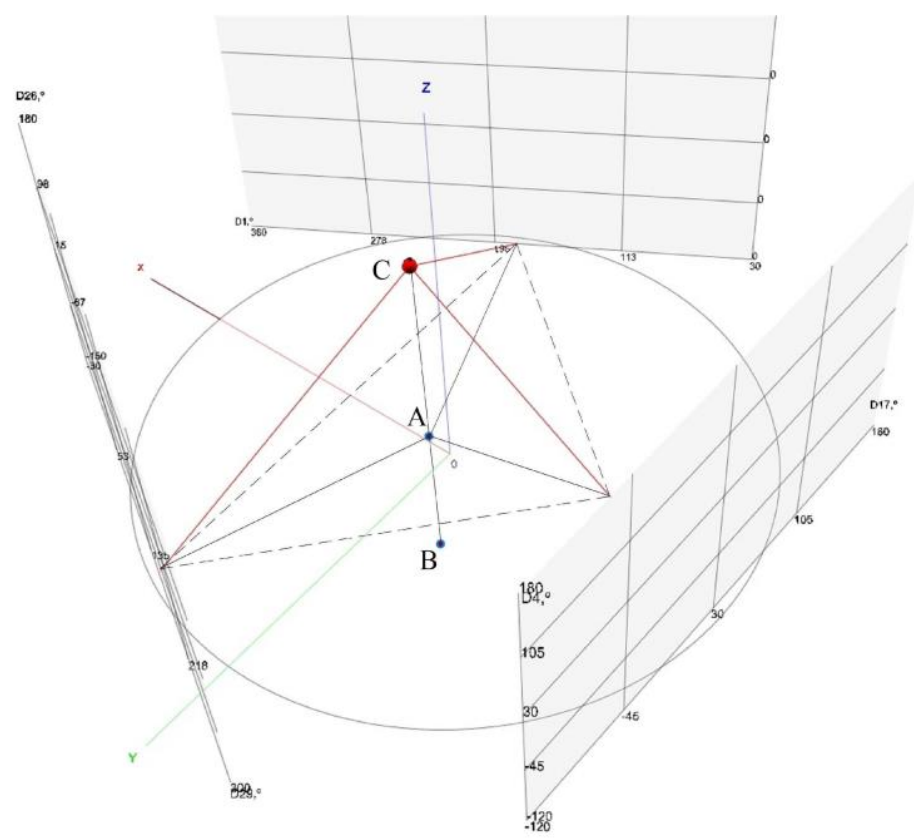

Figure 6. Display of $7 \mathrm{D}$ point by ball.

Further, point $A$ in Fig. 6 corresponds to the triangle centroid modeling a point in 6D space. Point B is the projection of the centroid onto the XOY plane of Exidiagraph. Point $C$ corresponds to the normalized position of the centroid in accordance with the actual value of the hyperfunction at a given point on the surface. For example, the points in this case are $7 \mathrm{D}$ points, considering their color. Fig.7 is an example of some $7 \mathrm{D}$ points (the hypersurface minima. The minima finding algorithm see in [9-11]) of hypersurface in Exidiagraph space. We presented these points as a set of balls. 


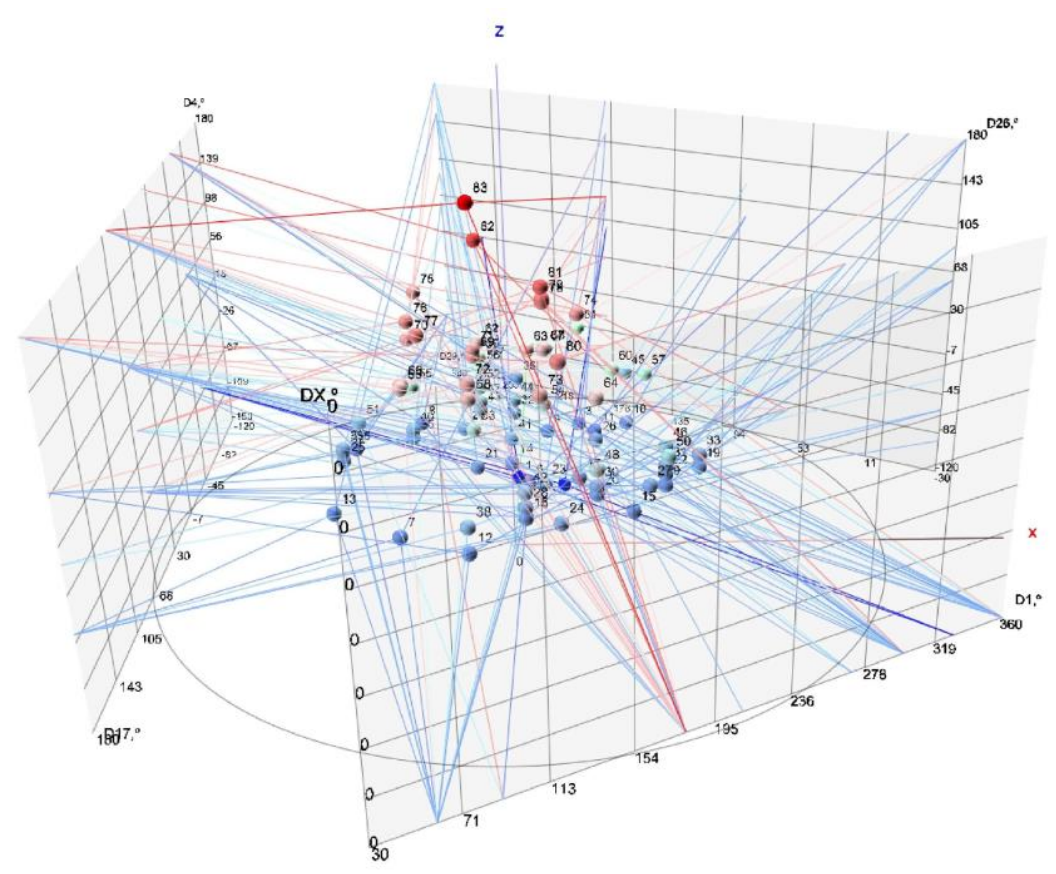

Figure 7. Display of some 7D hyper surface points by balls.

The described idea of multidimensional points visualization makes it possible to display a multidimensional surface defined in a table form in the Exidiagraph space. We called it an adapted hyper voxel model of multidimensional hypersurface in contrast to the model in Fig.5. The model is based on the concept of pseudo-voxel, which is an element of space up to $7 \mathrm{D}$ including. In this case, visualization considers the nature of hypersurface as a function in the form of a multidimensional table. In order to visualize a multidimensional table each cell of it is mapped to a hyper voxel. In its turn, each hyper voxel is visualized according to the scheme described in this section. The final visualization in Exidiagraph is shown in Fig.8. In fact, this visualization is very compact, the points in space are located more precisely according to the real values of the tabular hyperfunction, that provides a visually clearer perception of the hypersurface.

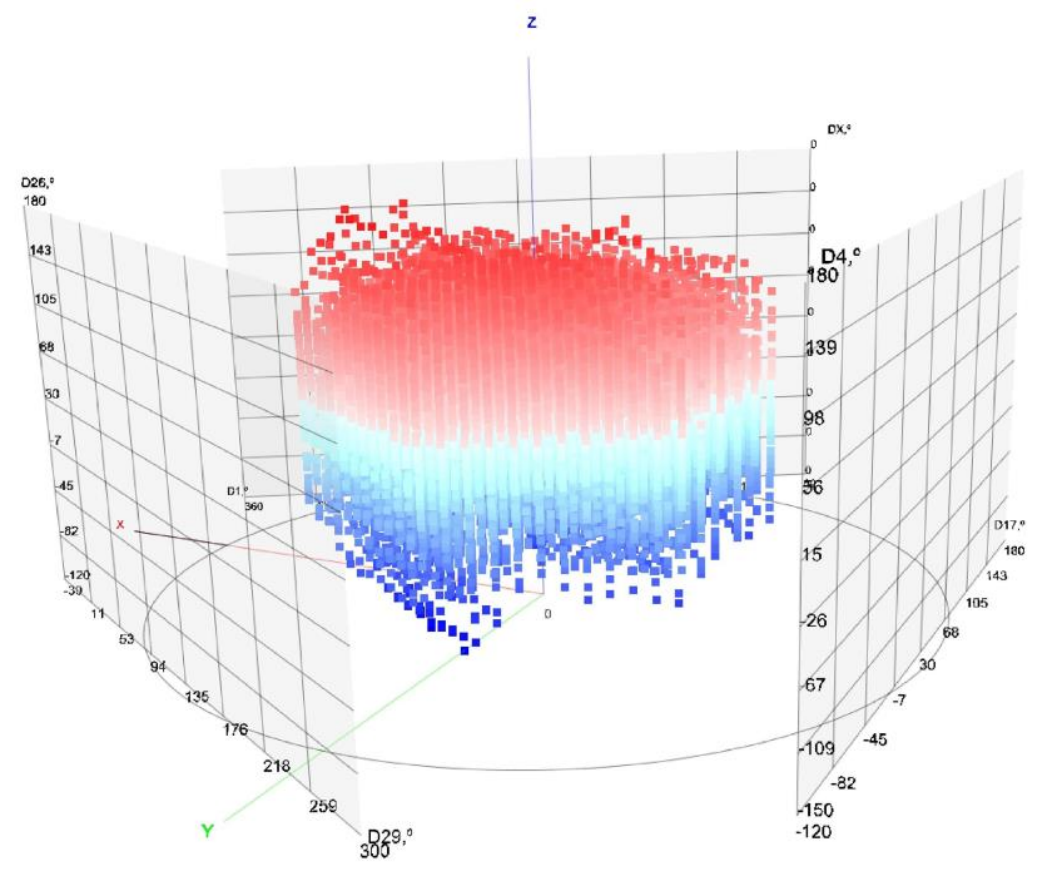

Figure 8. Display of 7D hyper surface by adapted hyper-voxels. 


\section{Finding the multidimensional hypersurface extrema}

Mathematically, the problem of finding the function extrema is related to finding such points of it, in which the first partial derivatives of functions go to zero, and the second partial derivatives are negative or nonnegative at the same time.

This issue requires finding such point $x$ that the scalar function $f\left(x_{1}, x_{2}, x_{3} \ldots x_{\mathrm{n}}\right)$ takes a value that is lower than at any neighboring point. For smooth functions, the gradient $g=\nabla f$ vanishes at the minimum. This problem is also known in mathematics as optimization problem. Optimization problem is a famous field of the science, engineering and technology. When solving optimization problem, it is necessary to calculate the global extremum (or its good approximation) of a function with multiple variables. The variables which define the optimized function can be continuous or discrete and, additionally, they often should satisfy certain constraints. Problems of NP-hard complexity class, which include optimization problems, are very difficult to solve. Therefore, traditional descent optimization algorithms are not suitable for their solution due to the local nature of the processed information [14]. In recent decades a lot of new algorithms for Global optimization problem solution have appeared. This led to success in a wide class of problem solution in various fields, such as computational chemistry and biology, computer science, economics, engineering design and others. But in general, there are no efficient methods available to minimize $n$-dimensional functions. The algorithms come from the initial assumption using a search algorithm that tries to move in an oblique direction. Algorithms using a gradient function minimize the one-dimensional line along this direction until the lowest point with a suitable tolerance is found. Then, the search direction is updated according to local information about the function and its derivatives, and the whole process is repeated until a true n-dimensional minimum is found. Algorithms that do not require a function gradient use a different approach. That is, the Nelder-Mead Simplex algorithm supports $n+1$ test parameter vectors as vertices of an $n$-dimensional simplex. The algorithm tries to correct the worst vertex of the simplex at each iteration using geometric transformations. Iterations continue until the total size of the simplex is sufficiently reduced. The problem seems to be very challenging for non-smooth or discrete functions. Actually, computational chemistry packages produce molecular energy values in the form of a large multidimensional table, depending on 2, 3, 4 or more variables, which correspond to a number of molecule variables. In this case, tabulated hyper surface has no analytic expression, which seriously complicates the search for its minima. In addition, the time for calculating energy critically depends on a number of variables, as well as on the calculation step for each given variable. With a larger number of variables and more condensed step, the calculation time can take up to several months of continuous calculation on super computing platforms. It complicates obtaining hypersurface extrema landscape with an acceptable reliability. For this problem solution the approach that contains two stages is developed and described in works [9-10].

\section{Conclusion}

As noted above, the described idea can be extended to cases of hypersurfaces with higher orders. The consecutive addition of extra planes with $2 \mathrm{D}$ coordinate systems to the Exidiagraph space allows displaying hypersurfaces of orders $7 \mathrm{D}, 8 \mathrm{D}$ and higher. There is still one drawback associated with the binding of each specific hyper voxel to coordinate systems. The problem is caused by hiding the hyper voxel communication lines as a result of necessity to avoid image overload. However, the disadvantage can be overcome by an interactive database of hypervoxel coordinates associated with the Exidiagraph model.

In conclusion, selecting a programming tool for the development of Exidiagraph model visualizer several platforms were analyzed and the Java language [15] together with its Java 3D extension [16] appeared to be the most suitable tools. The Internet technologies and the Java language have brought about a fundamental change in the way applications are designed and deployed. Java's "write once, run anywhere” model lessen the complexity and cost normally 
associated with producing software on multiple distinct hardware platforms. "Exidiagraph visualizer" has the Certificate of Federal Intellectual Property Service [17].

\section{Acknowledgement}

The work was supported by the Russian Foundation for Basic Research, RFBR grant № 1907-01024; and by Dr. B. Mez-Starck Foundation (Germany).

\section{References}

1. Wehrend S. and Lewis C. "A Problem-Oriented Classification of Visualization Techniques", Proceedings of the 1st IEEE Conference on Visualization '90, 1990, pp.139-143.

2. Etemadpour R. et al. Choosing Visualization Techniques for Multidimensional Data Projection Tasks: A Guideline with Examples. (2016, 166-186 pp 598. 166-186. 10.1007/9783-319-29971-6_9.

3. Keim D. A. and Kriegel H.-P. "Visualization Techniques for Mining Large Databases: A Comparison", IEEE Transactions on Knowledge and Data Engineering, vol.8, no.6, 1996 pp.923-938.

4. Bondarev A.E. On visualization problems in a generalized computational experiment / Scientific Visualization, 2019, volume 11, number 2, pages 156 - 162, http://doi.org/ 10.26583/sv.11.2.12

5. Bondarev A.E., Galaktionov V.A., Shapiro L.Z. Processing and visual analysis of multidimensional data / Scientific Visualization. V.9, № 5, c.86-104, 2017, DOI: http://doi.org/10.26583/sv.9.5.08

6. Mueller T.J. Flow Visualization by Direct Injection. Fluid Mechanics Measurements, Edited by Goldstein R.J, Hemisphere Pub. Co., 1983, 307-375 pp.

7. Rogers, David F., (2001). An introduction to NURBS: with historical perspective, - Morgan Kaufmann Publishers, ISBN 1-55860-669-6.

8. Foley J. D. et al, 1990. Computer Graphics: Principles and Practice. Addison-Wesley. ISBN 978-0-201-12110-O

9. Popov E., Batiukov A., Vogt N., Popova T., Vogt J. Visualization and Analysis of Molecular Potential Energy Surface (Pes) and Its Minima. IADIS International Conference Interfaces and Human Computer Interaction 2019 (part of MCCSIS 2019), Porto, 2019. pp. 411-415.

10. Popov, E. V., Batiukov, A. A., Vogt, N., Popova, T. P., \& Vogt, J. (2020). Visualization and Minima Finding of Multidimensional Hypersurface. In P. Isaias, \& K. Blashki (Eds.), Interactivity and the Future of the Human-Computer Interface (pp. 282-309). Hershey, PA: IGI Global. doi:10.4018/978-1-7998-2637-8.ch015

11. Popov E., Popova T., Batiukov A., Vogt N. Visualization of Multidimensional Hypersurface Extrema by Lumigraph (2020). Scientific Visualization 12.2: 1 - 8, DOI: 10.26583/sv.12.2.01

12. Götler S. et al, The Lumigraph. In Computer Graphics, Annual Conference Series (Proc. SIGGRAPH '96): 1996. pp. 43-54.

13. B. Shneiderman, “The Eyes Have It: A Task by Data Type Taxonomy of Information Visualization," presented at IEEE Symposium on Visual Languages '96, Boulder, CO, 1996.

14. Zhigljavsky A. A. ,1991 Theory of Global Random Search, Kluwer Academic.

15. Niemeyer P. et al, 2013. Learning Java, 4th Edition. A Bestselling Hands-On Java Tutorial. O'Reilly Media, Inc.

16. Selman, D. 2003. Java3D. Programming. - Manning Publications Co Austin, - 302 p.

17. Popov E.V., Batiukov A.A. Exi Diastaseon Grafima (ExiDiaGraph). Certificate of a computer program RU 2020612284, 19/02/2020. Application No. 2020611368/69 of 10/02/2020. 